\title{
Aneurysmal Bone Cyst of the Middle Cuneiform: A case report
}

\author{
by Al Kline, $\mathrm{DPM}^{1} \square$
}

\section{The Foot \& Ankle Journal 1 (6): 2}

The incidence of Aneurysmal bone cyst $(A B C)$ is rare. About 1\% of all primary bone tumors constitute these lesions. They are most common in the upper extremity and rarely are reported in the foot. A case is presented reporting $A B C$ of the middle cuneiform. To date, this appears to be the only documented case of $A B C$ to the middle cuneiform. Surgical presentation and treatment using AlloMatrix ® bone putty as a moldable composite for an small, uncontained defect is described.

Key words: Aneurysmal Bone Cyst, ABC, Allomatrix ${ }^{\circledR}$ bone putty. reproduction in any medium, provided the original work is properly cited. @The Foot \& Ankle Journal (www.faoj.org)

\begin{abstract}
Aneurysmal bone cysts (ABC's) in the foot are rare. This bone tumor is usually found in the upper extremity, along metaphyseal regions of bone. Its frequency of occurrence in the foot bones is about $3 \%$ compared to other bones of the body. ${ }^{1}$ ABC's represent about $1 \%$ of all primary bone tumors collectively. ${ }^{2}$ The etiology is unknown, although it is now commonly accepted that benign bone cysts are caused by trauma. These tumors are benign and are characterized by expansile compartmentalization within cancellous bone. The lesions consist of blood filled spaces filled with connective tissue septa containing bone or osteoid and osteoclastic giant cells. $^{2}$ Differential diagnosis will include giant cell bone tumor, telangiectatic osteosarcoma and angiosarcoma.
\end{abstract}

\footnotetext{
Address correspondence to: $\mathrm{Al}$ Kline, DPM

3130 South Alameda, Corpus Christi, Texas 78404.

1 Adjunct Clinical Faculty, Barry University School of Podiatric Medicine. Private practice, Chief of Podiatry, Doctors Regional Medical Center. Corpus Christi, Texas, 78411.
}

The cyst is increasingly painful as it begins to expand, and diagnosis will usually be confirmed by 'fluid-fluid' levels and compartmentalization seen on CT and MRI.

The cyst always begins within the cancellous bone and can erode through the cortex and expand to surrounding articular bone. However, this is a slow process and usually increased pain is associated with the cyst before it has a chance to erode through the cortex. The cyst is slow growing.

\section{Classification}

Classification of aneurysmal bone cysts are often staged. Campanicci, et al., described five distinct stages of Aneurysmal bone cyst formation. ${ }^{3}$ 


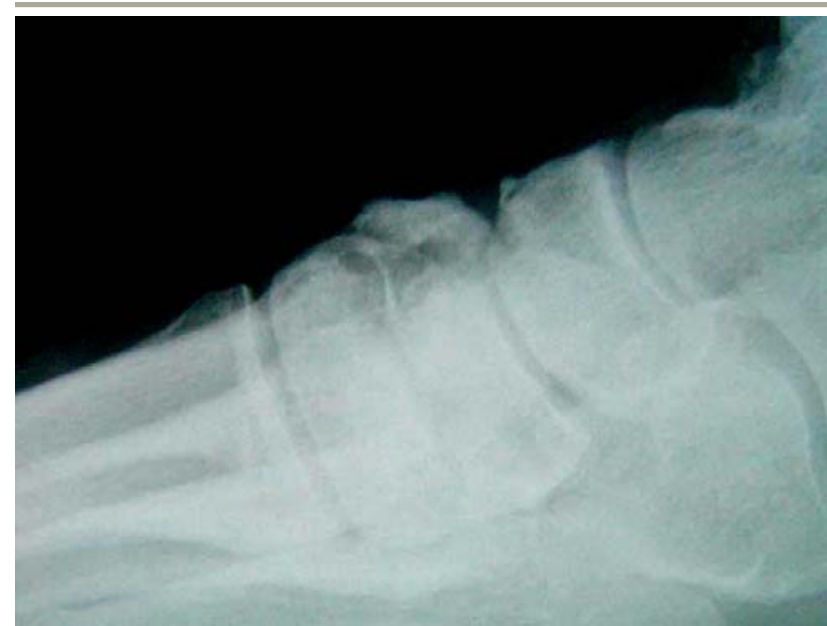

Figure 1 Expansile changes to the bone are noted along the middle cuneiform. Cystic changes appear to involve both the base of the $2^{\text {nd }}$ metatarsal and middle cuneiform.

Staging is based primarily on extent, size of cyst and its proximity to the cortex and soft tissue. Type $1 \mathrm{ABC}$ usually occupies the center of the bone with intact or slightly expanded profile. In long bones, it is usually situated along the metaphyseal region of bone. In Type $2 \mathrm{ABC}$, the cyst will occupy the entire width of the bone with an enlarged profile. These larger central cysts are primarily metaphyseal. In Type $3 \mathrm{ABC}$, the cyst is eccentric and interosseous with minimal expansion to the cortex. In Type $4 \mathrm{ABC}$, the cyst is now under the periosteum with superficial erosion of the cortex. In Type $5 \mathrm{ABC}$, the cyst has expanded through the cortex and invades the surrounding soft tissue. (Table 1)

\section{Case Report}

A 42 year old diabetic female presents to our office with a chief complaint of increased pain to the mid-tarsal region over the past year. There is no history of trauma. No allergies are reported. The patient is presently on medications for diabetes, hypertension and hypercholesterolemia.
Type 1: The cyst occupies the center of the bone with the profile either intact or slightly expanded. The lesion is usually metaphyseal in nature.

Type 2: The cyst occupies the entire width of bone and the profile is severely enlarged. They are termed "central cysts" and are primarily metaphyseal.

Type 3: The cyst appears as an eccentric, intraosseous lesion showing minimal expansion of the cortex.

Type 4: The cyst is subperiosteal with superficial erosion of the underlying cortex.

Type 5: The cyst has expanded into soft tissues and the cortex is destroyed.

Table 1 Campanicci staging of aneurysmal bone cysts.

The patient's medical history is also significant for anemia, end-stage renal disease and heart disease. Surgical history includes cataract replacement and hysterectomy.

Clinical evaluation reveals good pulses. On neurological examination, the patient has advanced diabetic neuropathy; however, there is still point tenderness over the mid-tarsal joint along the middle cuneiform just proximal to the second metatarsal. This coincides with palpatory pain to the middle cuneiform. Radiographic evaluation reveals expansile changes to the second cuneiform noted on lateral radiograph. (Fig. 1) MRI was ordered, revealing fluid-fluid levels on axial T1 imaging. (Figs. 2,3,4) Fat saturated post gadolinium T1 coronal axial study reveals high intensity changes within the superior half of the body of the cuneiform. (Figs. 5,6) Irregular cortical changes are consistent with fracturing of bone, and compartmentalization is seen in both T1 and T2 imaging. Closer examination of the inversion recovery sequencing or STIR images show cortical erosion extending along the proximal base of the second metatarsal base. (Figs. 7,8) This would classify the cyst as Campanicci Type 5, with expansion of the cyst through the cortex and into soft tissue. 


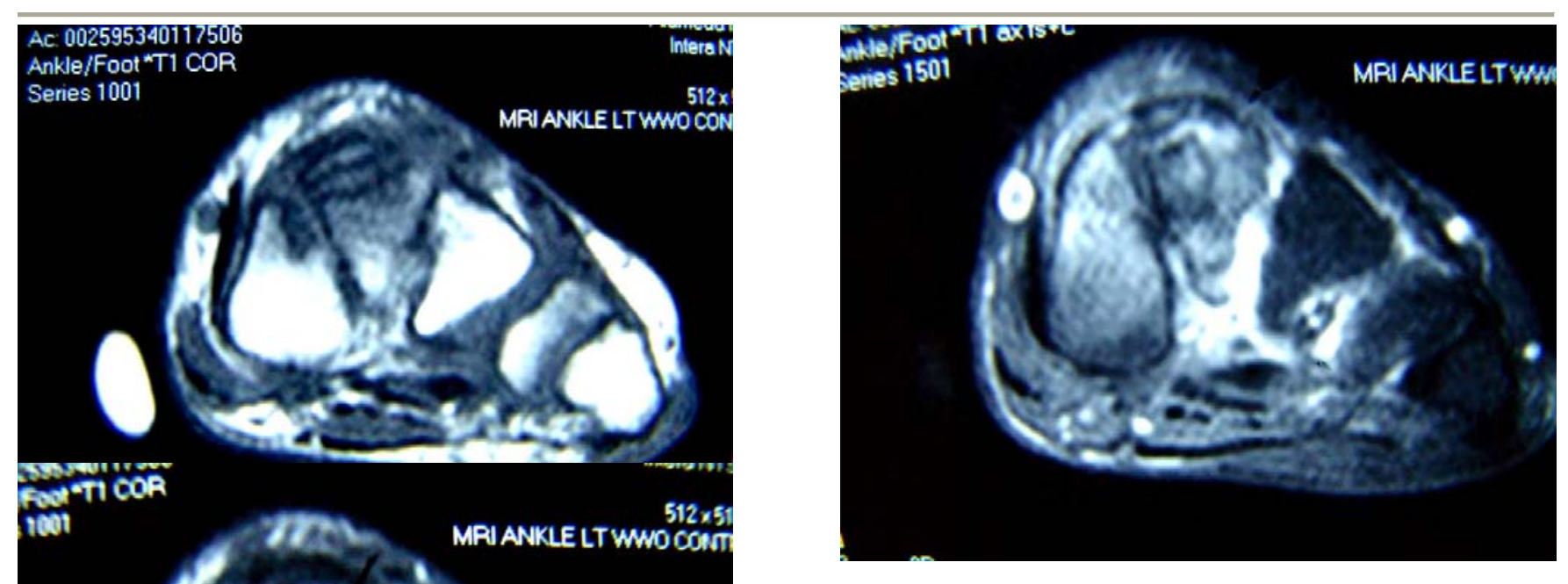

Figures 5,6 Fat saturated post gadolinium T1 images reveal fracturing and compartmental changes to the body of the middle cunieform. In larger bones, this would correspond to the 'soapbubble' effect often associated with the ABC.

Figures 2,3,4 T1 coronal/axial images reveal classic fluid-fluid within the superior pole of the middle cuneiform (Fig. 2). Significant cyst growth is noted along the main body of the middle cuneiform (Figs. 3,4). 


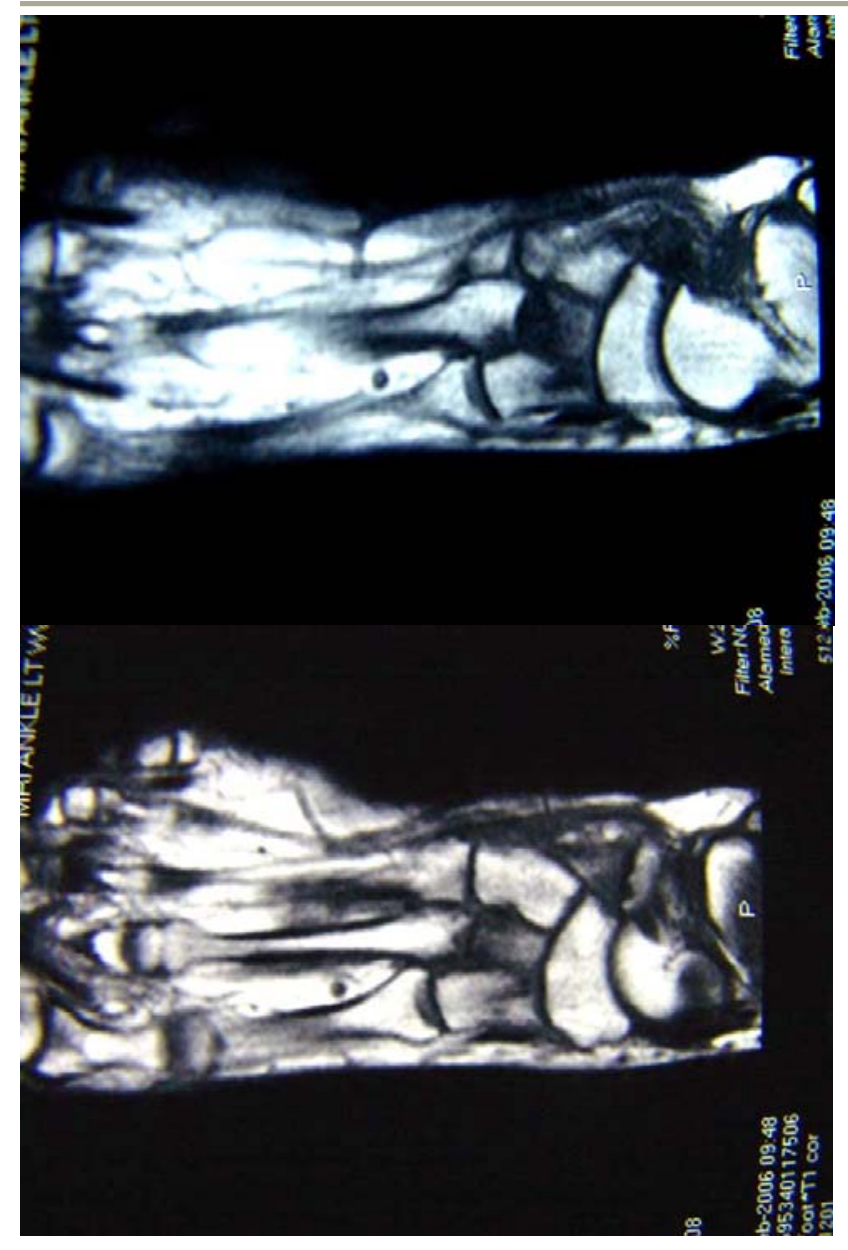

Figures 7,8 T1 images reveal extension of the cyst through the cortex of the middle cuneiform and base of the second metatarsal. This would characterize the lesion as a Campanicci Type 5 ABC.

A decision to schedule surgery was made and the patient was scheduled for cyst curettage with bone matrix therapy using AlloMatrix « bone putty. ${ }^{4}$

\section{Surgical Technique}

A curvilinear incision was made directly over the middle cuneiform. Once deepened to the bone structure of the cuneiform, a curette was used to identify and dorsally window the cyst.

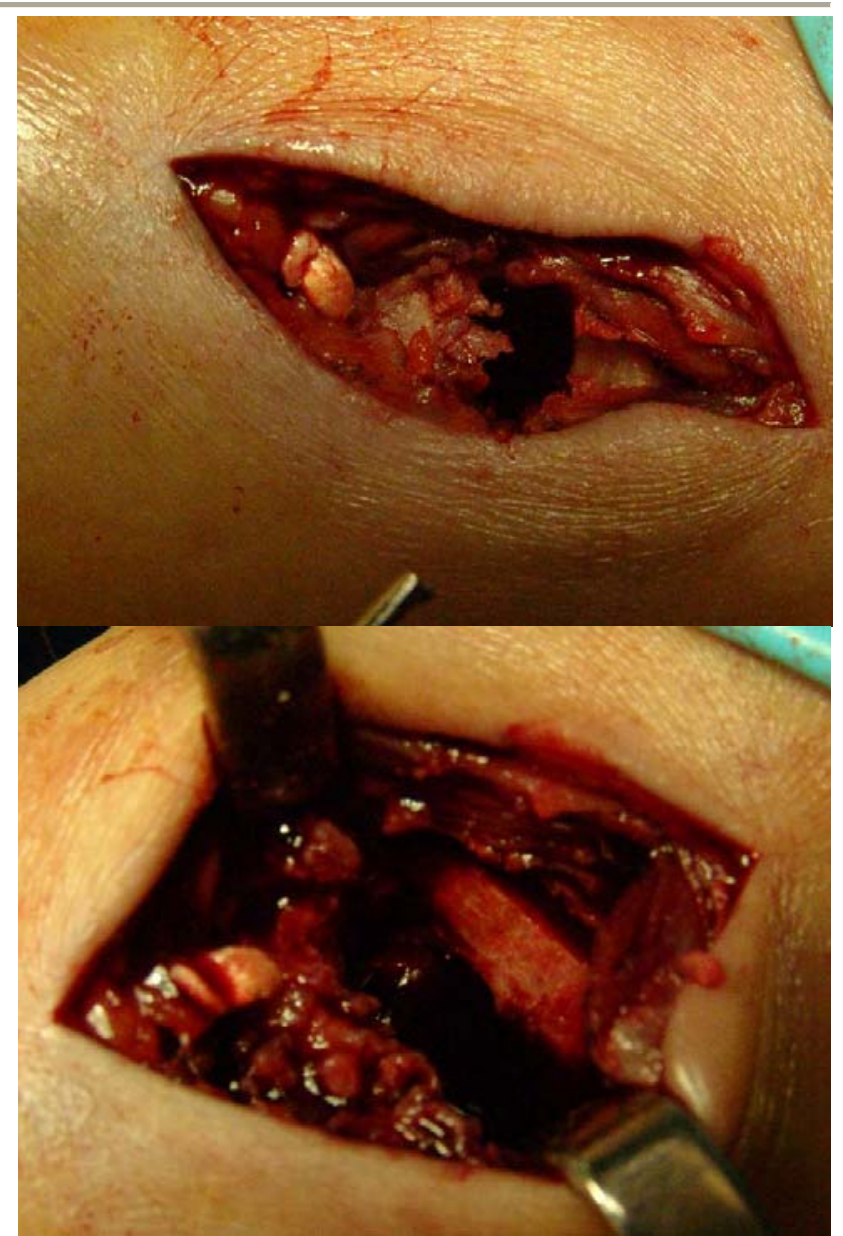

Figures 9,10 The dorsal cortex of the middle cuneiform and $2^{\text {nd }}$ metatarsal base is curettaged. A bone window is created revealing a soft cavity extending into the body of the middle cuneiform.

The entire cyst was evacuated to firm bone structure at every margin. A portion of the second metatarsal base was also curettaged. The cyst appeared to invade just the superiomedial aspect of the base of the second metatarsal. (Figs. $9,10)$ Material evacuated from the body of the cuneiform was sent to pathology. Pathology report revealed sections of decalcified specimen with fragments of bone and cartilage. Fragments of devitalized bone, marginated by cartilage and chondrocyte cloning with variable cellularity. 


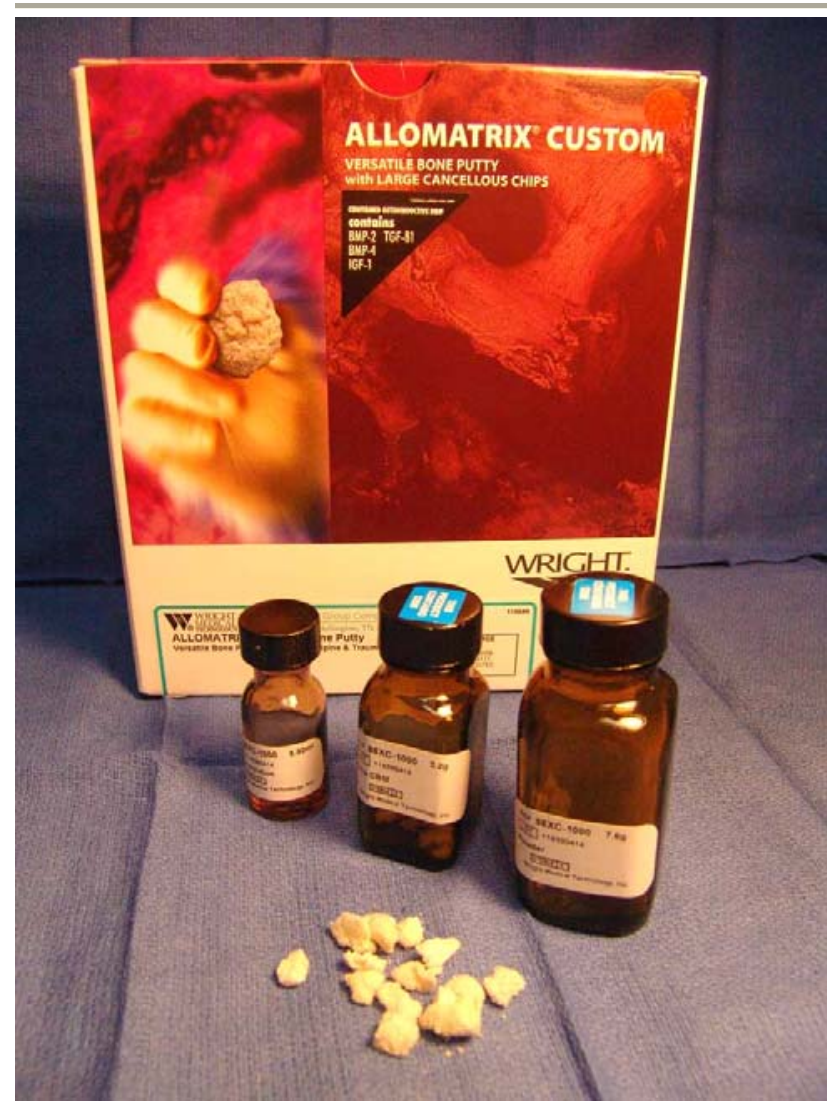

Figure 11 AlloMatrix ${ }^{\circledR}$ bone putty kit.

The material was serosanguineous in consistency. Areas of gelatinous-like material, deep red and purpuric in color were also evacuated from the body of the cuneiform.

The area and cavity were then lavaged with saline. AlloMatrix ${ }^{\circledR}$ bone putty (Fig. 11) was mixed and impacted into the void. (Figs. 12,13) The surgical site was then closed in a layered fashion. Postoperative radiographs show the defect filled with synthetic bone graft. (Figs. 14,15)

\section{Discussion}

In 1942, Jaffe and Lichtenstein first described $\mathrm{ABC}$ as a distinct entity when they discovered "a peculiar blood-containing cyst of large size." 5 Most ABC's will occur between the ages of 1030. ${ }^{1}$ Seventy-five percent of reported ABC's occur under the age of 20 , which would make this case even more unusual. There are variable techniques used to treat $\mathrm{ABC}$ 's.

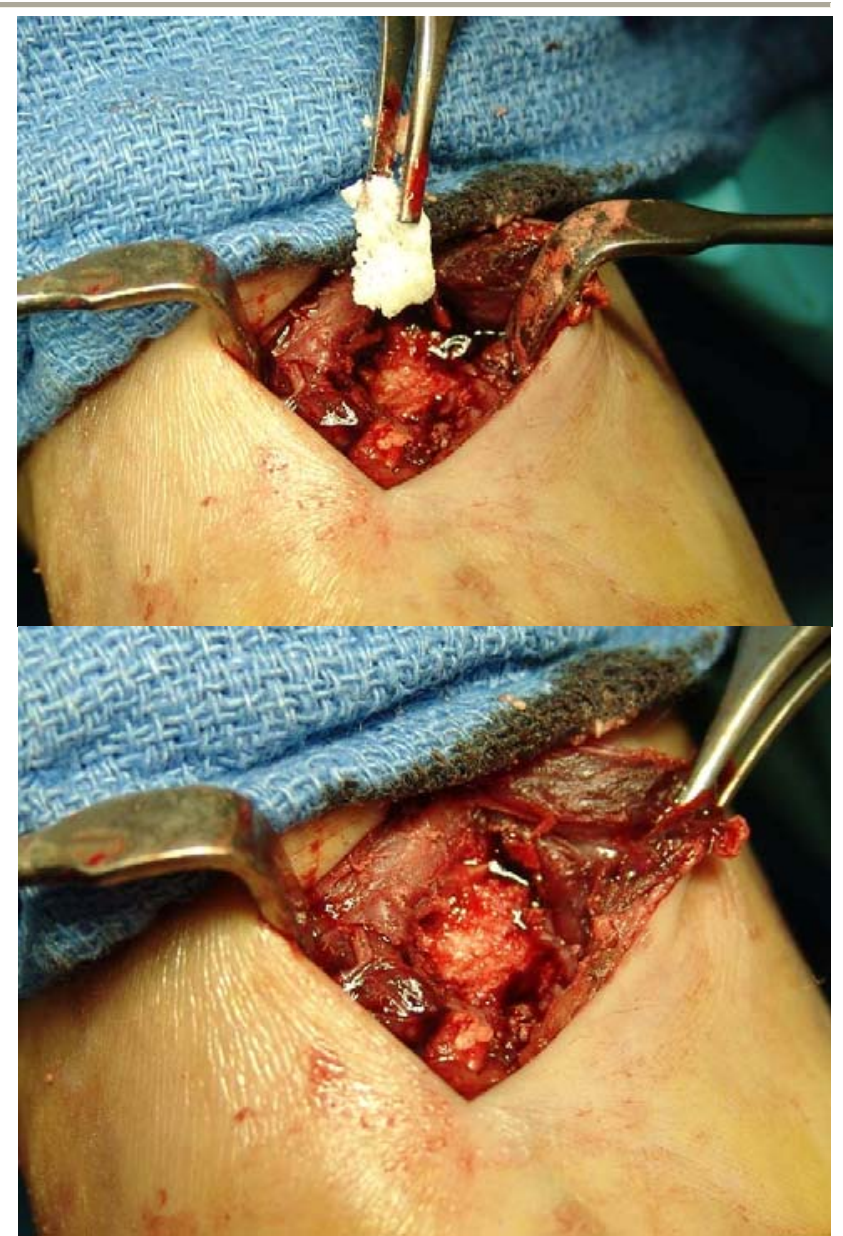

Figures 12,13 AlloMatrix ${ }^{\circledR}$ bone putty is mixed and inserted into the defect. The consistency of the putty can be varied by the amount of saline used to mix the substrate.

Some of these techniques include arterial embolization, intralesional injection, radionuclide ablation, local resection, en-bloc excision and intralesional removal.

Adjuvant therapies have also been attempted post resection including the use of liquid nitrogen and phenol. ${ }^{6}$ Once the cyst is evacuated, a number of various materials have also been used to fill the void. The cavity can be packed with either bone autograft, heterograft and even polymethyl methacrylate. After treatment, the incidence of reported recurrence has ranged from 10 percent and higher. This has prompted additional studies on adjuvant treatments such as cryotherapy. 


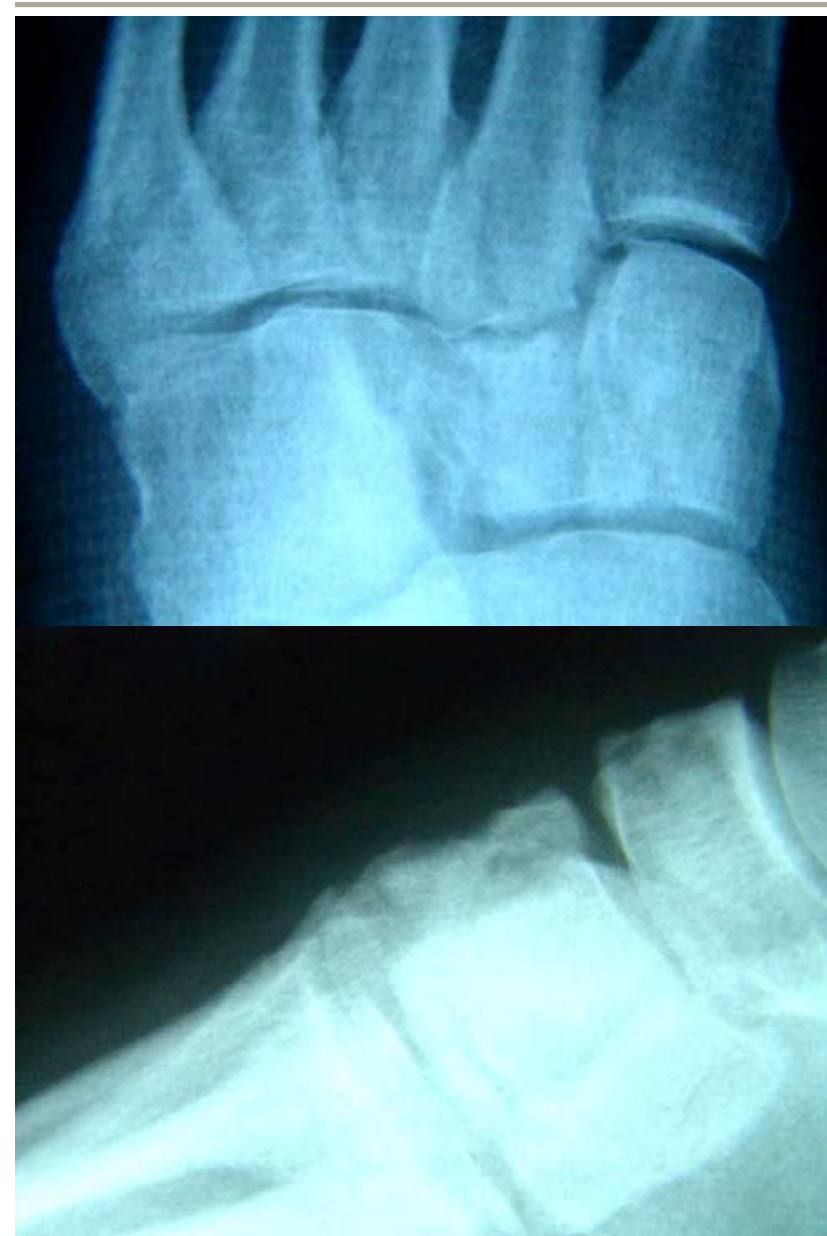

Figures 14,15 Post operative radiographs reveal AlloMatrix ${ }^{\circledR}$ bone putty filling the cyst defect.

In a landmark study by Gibbs, et al., forty patients treated with $\mathrm{ABC}$ excision by the same surgeon from 1976 to 1993 were reviewed. Thirty-four of the forty patients reviewed had curettage of bone using a high-speed burr. Of the twenty-two patients who had cancellous bone autogenous grafting, only $12 \%$ (4) had local recurrence. Of six patients who had resection of the cyst through its margin, none had recurrence. ${ }^{7}$ In 1997, Schreuder, et al., from the University Hospitals of Nijmegen and Groningen, in The Netherlands, reported their results on curettage with the adjunctive use of cyotherapy. Twenty-Six patients with twenty-seven ABC's were evaluated for complications and functional outcome after surgery. A mean follow-up of 47 months (19 to 154) was reported with only one local recurrence. They found similar results to those of marginal resection. When used in combination with bone grafting, they achieved bone consolidation in all of the patients. ${ }^{8}$

However, most of the past studies involve patients with large cysts of mostly long bones. Again, smaller bones such as cuneiforms, to this date, have not been reported. It was the author's choice to not use an adjuvant procedure after removal of the cyst.

In summary, this is a unique case of $A B C$ in a diabetic female in her fourth decade. She does not fit the profile for development of this lesion, especially in the foot. However, due to her diabetes and associated neuropathy, it could be surmised that neuropathy and trauma may play an important role in the development of benign bone cysts.

\section{References}

1. Anand, M.K., Wang, E.A. Aneurysmal Bone Cyst. eMedicine, Jan, 2007

2. Duke Orthopaedics: Wheeless' Textbook of Orthopaedics, Aneurysmal Bone Cyst, Online article, Jan 2007

3. Campanacci, M. et al: Unicameral and aneurismal bone cysts: A study of 416 UBC and 198 ABC. Clin Orthop 204:25, 1986

4. Wright Medical: AlloMatrix® Bone Putty with DBM\&Cancellous Chips.

5. Jaffe, H.L., Lichtenstein, L.: Solitary unicameral bone cyst with emphasis on reoentgen picture, the pathologic appearance and the pathogensis. Arch Surg 44: 1004-1025, 1942

6. Eastwood, B., Biggs, H.K.: Aneurysmal Bone Cyst, eMedicine Online article, 2007

7. Gibbs, C.P. et al: Aneurysmal Bone Cyst of the Extremities. Factors Related to Local Recurrence After Curettage with a High-Speed Burr. JBJS 81:1671-1688, 1999. [Free online]

8. Schreuder, H.W., et al: Aneurysmal Bone Cysts Treated by Curettage, Cryotherapy and Bone Grafting, JBJS (UK), 79-B, No. 1, January, 1997. 